\title{
Pupillary correlates of lapses of sustained attention
}

\author{
Nash Unsworth $^{1}$ - Matthew K. Robison ${ }^{1}$
}

Published online: 1 April 2016

(C) Psychonomic Society, Inc. 2016

\begin{abstract}
The current study examined the extent to which pupillary responses (both pretrial baseline and phasic responses) would accurately track lapses of attention as predicted by theories of locus coeruleus norepinephrine (LC-NE) functioning. Participants performed a sustained attention task while pupil responses were continuously recorded. Periodically during the task, participants were presented with thought probes to determine if they were on or off task. The results suggested the pupillary responses accurately distinguished on from off-task states. Importantly, pretrial baseline pupil responses distinguished different types of lapses of attention, with inattentive and mind-wandering states being associated with small pretrial baseline pupil diameters on average and distracted states being associated with larger pretrial baseline pupil diameters on average compared to focused states. These results support the notion that pupil diameter is sensitive to different types of lapses of attention which may be associated with different LC-NE modes.
\end{abstract}

Keywords Cognitive control $\cdot$ Norephinephrine $\cdot$ Attention

The ability to focus and sustain attention on task-relevant information is a critically important skill that is needed in a host of everyday activities. Despite the importance of focusing and sustaining attention on goal-relevant information, sometimes the attention system falters, leading to lapses. Understanding these lapses, whereby attention is disengaged from the current

Nash Unsworth

nashu@uoregon.edu

1 Department of Psychology, University of Oregon, Eugene, OR 97403, USA task and focused on other external distracting stimuli or internal thoughts (daydreaming), is important for understanding how and when attentional processes falter in both the laboratory and in real-world situations (Reason, 1984). For example, the ability to focus attention is needed in a host of activities where any lapses of attention could result in unwanted outcomes, such driving accidents, lower academic performance, and failures to spot weapons during baggage screening (Reason, 1990; Unsworth, McMillan, Brewer, \& Spillers, 2012). Gaining a better understanding of fluctuations in attention is important for understanding the attentional system more broadly and for predicting when and for whom attention failures are most likely.

\section{Regulating Attentional State}

Theoretically, cognitive control processes are needed to ensure active goal maintenance leading to task-appropriate behaviors. Prominent theories of cognitive control suggest that cognitive control is implemented by the prefrontal cortex in situations where task goals need to be actively maintained and dynamically updated (Miller \& Cohen, 2001). Accordingly, top-down attention control processes are needed to maintain task goals and bias responding so that the appropriate behavior is executed. In many situations, participants will have to actively maintain a novel task goal that is in direct opposition to prepotent response tendencies (Roberts \& Pennington, 1996). If there is a failure of active goal maintenance, then it is likely that prepotent response tendencies will guide behavior, leading to goal neglect and the execution of the incorrect response (Duncan, 1995). In situations when attention is tightly focused on the task goal, performance will be both fast and accurate. However, if attention is not tightly focused on the task goal, goal neglect can occur. Thus, any lapses in attention will result in prepotent responses guiding behavior and hence the 
occurrence of fast reflexive errors (Unsworth, Schrock, \& Engle, 2004) or much slower than normal response times (Unsworth, Redick, Lakey, \& Young, 2010).

Recent research suggests that the locus coeruleus norepinephrine system (LC-NE) may be particularly important in modulating prefrontal cortex representations based on attentional control demands (Cohen, Aston-Jones, \& Gilzenrat, 2004). The LC is a brainstem neuromodulatory nucleus that is responsible for most of the NE released in the brain and it has widespread projections throughout the neocortex (Berridge \& Waterhouse, 2003). Generally the LC-NE system has been associated with general functions such as the sleepwake cycle and overall arousal levels. More recently neurophysiological and computational modeling studies (AstonJones \& Cohen, 2005; Aston-Jones, Iba, Clayton, Rajkowski, \& Cohen, 2007; Usher, Cohen, ServanSchreiber, Rajkowski, \& Aston-Jones, 1999) have suggested that the LC-NE system is important for regulating the balance between exploitation (maintaining attention on the current task) and exploration (task disengagement). A great deal of recent research (see Aston-Jones \& Cohen, 2005; Berridge \& Waterhouse, 2003, for reviews) suggests that there is an inverted-U relationship between LC tonic (baseline) activity and performance on various cognitive tasks consistent with the Yerkes-Dodson curve (Yerkes \& Dodson, 1908). Specifically, it is assumed that when tonic LC activity is low (hypoactive mode), individuals are inattentive and nonalert, leading to poor behavioral performance and little to no phasic LC activity in response to task-relevant stimuli. As tonic LC activity increases to an intermediate range (phasic mode), attention becomes more focused, LC phasic activity increases for target stimuli, and behavioral performance is optimal. However, as tonic LC activity increases further, the individual experiences a more distractible attentional state, leading to task disengagement, lowered LC phasic activity, and a reduction in behavioral performance. Intracranial recordings in rats and monkeys and psychopharmacological studies in animals and humans provide evidence in support of the notion of an inverted-U relationship between the LC-NE system and behavioral performance (Aston-Jones \& Cohen, 2005). Collectively this research suggests that the LC exhibits fluctuations between these various modes/states during simple attentional tasks, and these fluctuations are linked to fluctuations in behavioral performance. As such, prior research suggests that LC-NE system is important in regulating the current attentional state and that fluctuations in attentional state correspond to fluctuations in tonic LC activity.

\section{Pupil Diameter and the LC-NE System}

A potential candidate as a psychophysiological marker for changes in attentional state is pupil diameter. Much prior research has shown that the pupil dilates in response to the cognitive demands of a task (Beatty, 1982a). For example, Hess and Polt (1964) demonstrated that the pupils dilated as a function of problem difficulty in a mental multiplication task with higher peak dilations for the hardest problems. Similarly, Kahneman and Beatty (1966) demonstrated that pupillary dilation increased as more items were required for recall in a standard short-term memory task (see also Unsworth \& Robison, 2015). These effects reflect task-evoked pupillary responses (TEPRs) in which the pupil dilates relative to baseline levels due to increases in cognitive processing load. A number of studies have demonstrated similar TEPRs in a variety of tasks (see Beatty \& Lucero-Wagoner, 2000, for a review). These and other results led Kahneman (1973) and Beatty (1982a) to suggest that TEPRs are a reliable and valid psychophysiological marker of cognitive effort or processing load.

More recently, research has suggested that pupil dilations are indirectly related to the functioning of the LC-NE system (Aston-Jones \& Cohen, 2005; Gilzenrat et al., 2010; Joshi Cohen, Servan-Schreiber, Rajkowski, \& Aston-Jones, 2016). Specifically, Aston-Jones and Cohen (2005; based on results from Rajkowski, Kubiak, \& Aston-Jones, 1993) demonstrated a modest correlation between baseline pupil diameter and baseline firing rate of an LC neuron in a monkey (see also recent work by Joshi et al., 2016). According to Aston-Jones and Cohen (2005; see also Gilzenrat et al., 2010; Jepma \& Nieuwenhuis, 2011; Joshi et al., 2016), pupil diameter provides an indirect index of the LC modes such that in the hypoactive mode overall baseline pupil diameter is small. In the tonic LC mode, overall baseline pupil diameter is relatively large. Finally, in the LC phasic mode, overall baseline pupil diameter tends to be at intermediate levels. Collectively, this work suggests that baseline pupil diameter should provide an indirect index of LC-NE functioning. For example, prior research has demonstrated that drugs such as modafinil increase central NE, leading to increases in subjective alertness and increases in baseline pupil diameter (Hou, Freeman, Langley, Szabadi, \& Bradshaw, 2005). Drugs such as clonidine decrease central NE, leading to lowered subjective alertness and lower baseline pupil diameter (Hou et al., 2005). Furthermore, recent neuroimaging work has shown that activity in the LC is correlated with changes in pupil diameter (Alnaes et al., 2014; Murphy, O'Connell, O'Sullivan, Robertson, \& Balsters, 2014). Thus, there is a correlation between baseline pupil diameter and baseline firing rate of LC neurons, and drugs that increase or decrease central NE levels lead to increases or decreases in baseline pupil diameter. This work suggests that baseline pupil diameter can be used as an indirect reporter variable of LC-NE functioning.

Although prior work is suggestive of a link between LCNE functioning and pupil diameter, it should be acknowledged that this relation is correlational in nature and it could 
be due to the fact that both the LC and the sympathetic nervous system are linked via a third system. Specifically, Nieuwenhuis, de Geus, and Aston-Jones (2011) suggested that the LC-pupil link is not due to a direct causal pathway but rather is due to the fact that both the LC and the sympathetic nervous system are controlled by the nucleus paragigantocellularis. The influence of NE pharmacological manipulations on baseline pupil diameter could thus be due to the influence of lower medullary NE cell groups rather than the LC directly. It is also possible that pupillary changes reflect drug effects on the peripheral (rather than the central) nervous system, where NE is an important neurotransmitter. More definitive work is needed to establish the relationship between the LC-NE and pupil diameter. For now, pupil diameter can be used as an indirect measure of LC-NE functioning, while acknowledging that the relation could be due to a third variable (the nucleus paragigantocellularis).

Several recent studies have begun to explore whether pupil diameter provides an index of LC-NE functioning and changes in control state. For example, using a similar auditory detection task as that used by Rajkowski et al. (1993) to examine the relation between pupil diameter and LC tonic activity, Gilzenrat et al. (2010) found that trials that were preceded by large baseline pupil diameters were associated with poorer performance indicative of lapses of engagement corresponding to the LC tonic mode. Trials that were preceded by smaller baseline pupil were associated with better task performance indicative of task engagement corresponding to the LC phasic mode. Corroborative results were found in their additional experiments, suggesting that pupil diameter tracks changes in control state related to LC activity. Furthermore, Murphy et al. (2011) found an inverted-U relationship between baseline pupil size and performance on an auditory oddball task such that performance (in particular, variability in reaction time) was worse when baseline pupil was very small or very large, but performance was best at intermediate baseline levels. Murphy et al. suggested that baseline pupil diameter not only provides an index of the tonic and phasic modes (the upper and intermediate ends of the LCNE curve) but also provides an index of low levels of alertness and arousal (the lower end of the curve) and suggested that when examining links between pupil diameter and LC activity, the entire curve must be taken into account. This is consistent with prior research, which has consistently shown that under conditions of fatigue or low levels of alertness, baseline pupil diameter is smaller and more variable than when alert (Hou et al., 2005; Morad, Lemberg, Yofe, Dagan, 2000). Likewise, Kristjansson et al. (2009) found that baseline pupil diameter was much smaller on trials preceding very slow reaction times (indicative of lapses of attention) on a vigilance task compared to trials where reaction time was close to the mean. Kristjansson et al. suggested that fluctuations in alertness resulted in variable reaction times and that baseline pupil diameter provides an index of changes in alertness. Collectively, these results suggest that baseline pupil diameter provides an index of LC-NE functioning and can be used to track fluctuations in attentional control state.

\section{Thought Probing and Attentional State}

The aforementioned work suggests the importance of maintaining goal-relevant information in an active state to ensure accurate responding and further suggests that periodic lapses in attention or task engagement can lead to deficits in behavioral performance. Furthermore, prior research has suggested that baseline pupil diameter may provide an index of changes in attentional state. Another way of examining changes in attentional state is to simply ask participants what their current attention state is. Prior research has suggested that participants' self-reports of their attentional state provides important and useful information for understanding changes in attentional state (Smallwood \& Schooler, 2006). Specifically, a number of studies have utilized thought-probe techniques in which, periodically, during a prolonged attention task, participants are probed and are required to report whether their attention was currently focused on task or whether they were thinking of things unrelated to the task (i.e., mind-wandering). This research has consistently found that not only do participants report extensive off-task thoughts during attentional tasks, but also these self-reports of off-task thoughts are correlated with actual performance, such that self-reports of off-task thoughts are associated with lower levels of performance (McVay \& Kane, 2012b; Schooler, Reichle, \& Halpern, 2005). Additionally, rates of off-task thoughts have been shown to be associated with very slow reaction times, which have long been seen as indicators of lapses of attention (McVay \& Kane, 2012a; Unsworth et al., 2010). Prior work suggests that the probe techniques for examining variation in attentional state have been shown to be both reliable and valid and have demonstrated the importance of examining fluctuations in attentional state during a number of tasks and situations.

Most prior research that has used thought probe techniques has focused on whether participants are focused on the current task or whether they are mind-wandering. However, when participant's attention is not on the current task it is not always because they are mind-wandering. Rather, it is possible that sometimes participants engage in off-task thoughts about external distractors as well as internal distractors (i.e., mindwandering). Stawarczyk, Majerus, Maj, Van der Linden, and D'Argembeau (2011) introduced a novel experience sampling method to distinguish the different varieties of off task thoughts. Specifically, Stawarczyk et al. used a thoughtprobe technique in which participants were not simply instructed to indicate if they were on or off task, but rather participants had to indicate if they were on task, if they were 
experiencing task-related interference (interfering thoughts related to the appraisal of the current task such as worrying about performance), if they were distracted by external stimuli, or if they were mind-wandering. Implementing this technique in a sustained attention task, Stawarczyk et al. (2011) found that roughly $20 \%$ of the responses to the thought probes were external distractions and roughly $21 \%$ were mind-wandering. Furthermore, when participants reported that they experienced either external distraction or mind-wandering, performance was worse than when participants reported that they were focused on the task. In another recent study, we (Unsworth \& McMillan, 2014a) had participants perform a variety of attention control tasks, and participants responded to the same thought probes as used Stawarczyk et al. (2011). We found that participants who reported more lapses of attention (i.e., more mind-wandering and more external distraction) also tended to have more variable reaction times and more slow reaction times (see also McVay \& Kane, 2012a). Thus, off-task thinking can be directed towards internal thoughts and concerns (mind-wandering) or toward external stimuli unrelated to the task at hand (external distraction), and both of these can lead to lapses in performance. Finally, it is also likely that when participants report that they are not focused on the current task at hand, that sometimes they are simply inattentive (nonalert) and are not directing their thoughts to internal concerns or to external stimuli, but rather they are simply zoning out or experiencing mind-blanking (Ward \& Wegner, 2013), leading to lower behavioral performance (Unsworth \& McMillan, 2014b). Thus, thought-probe techniques can be used to examine fluctuations in attentional state and be used to classify various attentional states (i.e., focused, inattentive, mind-wandering, and/or distracted).

\section{The Current Study}

Despite initial research examining fluctuations in attention and control state with pupillary responses, more research is needed to better determine the extent to which fluctuations in various attentional states can be tracked accurately with changes in pupillary dilations and the extent to which these fluctuations correspond to subjective changes in attentional state. To our knowledge, only three prior studies have examined a potential link between changes in baseline pupil diameter and subjective attentional state by examining differences between on- and off-task states while simultaneously measuring baseline pupil diameter (Franklin, Broadway, Mrazek, Smallwood, \& Schooler, 2013; Grandchamp, Braboszcz, \& Delorme, 2014; Mittner et al., 2014). In one of these studies (Franklin et al., 2013) the authors found that off-task reports (thought to reflect mind-wandering) were associated with larger baseline pupil diameters than were on-task reports. However, both Grandchamp et al. (2014) and Mittner et al.
(2014) found that off-task reports were associated with smaller baseline pupil diameters than were on-task reports. Thus, there are clear conflicting results in the literature. One limitation with this prior research is that participants were simply asked if their current thoughts were on or off task. As noted previously, this simple dichotomous response ignores the many varieties of attentional lapses (e.g., inattentive, mind-wandering, distracted) and thus, it is unclear how pupil diameter is related to different attentional states.

It should be noted that other studies have examined the link between pupil diameter and attentional state, but these studies did not simultaneously measure pupil diameter and attentional state at the same time (Kang, Huffer, \& Wheatley, 2014; Smallwood, Brown, Tipper, et al., 2011; Smallwood, Brown, Baird, et al., 2012). Specifically, Smallwood et al. (2011) and Kang et al. (2014) demonstrated larger baseline pupil diameters in a choice reaction time task than in a working memory task and suggested that the larger baseline pupil diameters reflected more mind-wandering. In a separate experiment, Smallwood et al. (2011) demonstrated that more mind-wandering occurred in the choice reaction-time task than in the working memory task, but unfortunately they did not simultaneously measure both pupil diameter and attentional state in the same experiment, so it is unclear whether baseline pupil diameter is larger or smaller during a mind-wandering episode. In a similar vein, Smallwood et al. (2012) measured pupillary responses during a vigilance task but measured mind-wandering after the task with a questionnaire. Smallwood et al. found that individuals who reported more mind-wandering had larger pupils than individuals who reported less mind-wandering. Although there was a positive relation between pupil diameter and mind-wandering, again, pupil diameter and attentional state were not measured at the same time, and there is no indication for what the relation is within subjects (rather than between subjects). Thus, the more evidence is needed to examine the relation between pupillary responses and attentional state.

To examine whether baseline pupil diameter accurately tracks different attentional states, in the current study we had participants perform a sustained attention task (the psychomotor vigilance task; Dinges \& Powell, 1985). This task has long been used as a measure of sustained attention to examine how changes in alertness affect vigilant attention (Dorrian, Rogers, \& Dinges, 2005). Additionally, factors such as sleep deprivation tend to amplify these effects (Dorrian et al., 2005) and the slowest RTs in this task have been found to be linked to greater activity in the default-mode network (Drummond et al., 2005). Recent work also suggests that performance on this task correlates with performance on other attentional control tasks, including the Stroop task, flankers, antisaccade, and the sustained attention to response task (e.g., Unsworth \& 
McMillan, 2014a). Thus, there is good evidence that the slowest reaction times in this task provide an index of lapses of attention. During the psychomotor vigilance task, participants were periodically presented with thought probes asking whether they were on task, thinking about their performance on the task, distracted by external information, mind-wandering, or simply inattentive and not alert. Throughout the task participants' pupils were continuously measured. Building on prior research, it is assumed that baseline pupil diameter should provide an index of when individuals are focused on a task and when they are experiencing different types of lapses of attention. Specifically, Lenartowicz, Simpson, and Cohen (2013) suggested a landscape of attentional lapses wherein when arousal is low and attention is focused internally, mindwandering occurs. When arousal is low and attention is focused externally, attentional capture from bottom-up sources is expected to occur. When arousal is high and attention is focused internally, this can lead to internal distraction in the form of ruminations and racing thoughts. When arousal is high and attention is focused externally, this can lead to external distraction, resulting in oversensitivity to external stimuli. Thus, Lenartowicz et al. (2013) suggest that the type of lapse of attention is determined by arousal levels from the LC-NE system and by whether attention is directed to external stimuli or to internal thoughts.

In terms of pupil diameter, this suggests that baseline pupil diameter should provide a means to track different types of lapses of attention. When baseline pupil diameter is small participants should report being inattentive/ nonalert (corresponding to low tonic LC activity and low arousal), resulting in lower task performance and more lapses of attention. At intermediate baseline pupil diameters, participants should be focused on the task at hand (corresponding to the LC phasic mode and optimal arousal levels), leading to good task performance. Finally, when baseline pupil diameter is relatively large, participants should report that they are distracted and disengaged from the task (corresponding to the LC tonic mode and over arousal) leading to lower task performance and increases in lapses of attention. Furthermore, we examined time on task effects to determine how pupil diameter changes as a function of time on task and whether this is related to corresponding changes in performance and attentional state. Specifically, as time on task increases, we expect that performance should worsen (i.e., the vigilance decrement), and participants should report being unfocused and off task more (i.e., more mind-wandering and inattentiveness), and pupil diameter should correspond to changes in attentiveness. Thus, it is predicted that there should be a link not only between pupil diameter and performance but also between pupil diameter and different attentional state reports.

\section{Method}

\section{Participants}

Participants were 39 undergraduate students recruited from the subject pool at the University of Oregon. Based on prior pupillometry work, which suggested a medium to large effect size (Franklin et al., 2013; Grandchamp et al., 2014; Mittner et al., 2014) we determined that a minimum sample size of 35 participants would be sufficient to find a medium effect size, with power of .80 and alpha set at .05 (two tailed). Participants were between the ages of 18 and 35 and received course credit for their participation. Data from two participants were excluded from analyses because of data collection problems with the eye tracker, leaving a final sample of 37 participants.

\section{Procedure}

Participants were tested individually in a dimly lit room. Pupil diameter was continuously recorded binocularly at $120 \mathrm{~Hz}$ using a Tobii T120 eye tracker. Participants were seated approximately $60 \mathrm{~cm}$ from the monitor. After providing informed consent and after calibrating the eye tracker, participants performed a variant of the psychomotor vigilance task (Dinges \& Powell, 1985). Stimuli were presented on the Tobii T120 eye tracker 17 -in. monitor with a $1,024 \times 768$ screen resolution. As shown in Figure 1, in this task participants were first presented with a row of five black fixation crosses in the middle of the screen on a gray background for 2,000 ms. Participants were then presented with a row of zeros in blue Arial 24-point font (visual angle $1.21^{\circ}$ ) in the center of the screen, and after a variable wait time (equally distributed from 2 to $10 \mathrm{~s}$ in in $500 \mathrm{~ms}$ increments) the zeros began to count up in $17-\mathrm{ms}$ intervals from $0 \mathrm{~ms}$. The participants' task was to press the spacebar as quickly as possible once the numbers started counting up. After pressing the spacebar, the RT was left on screen in red for $1 \mathrm{~s}$ to provide feedback to the participants (shown in bold in Figure 1). Following feedback ,a $500 \mathrm{~ms}$ blank screen was presented and then either the next trial started or participants were presented with a thought probe. Participants performed 160 trials, and the experiment lasted approximately $30 \mathrm{~min}$. Thirty thought probes were randomly presented after roughly $19 \%$ of the trials equally distributed across blocks.

\section{Thought Probes}

During the task, participants were periodically presented with thought probes asking them to classify their immediately preceding thoughts. The thought probes asked participants to press one of five keys to indicate what they were thinking just prior to the appearance of the probe. Specifically, participants saw the following: 


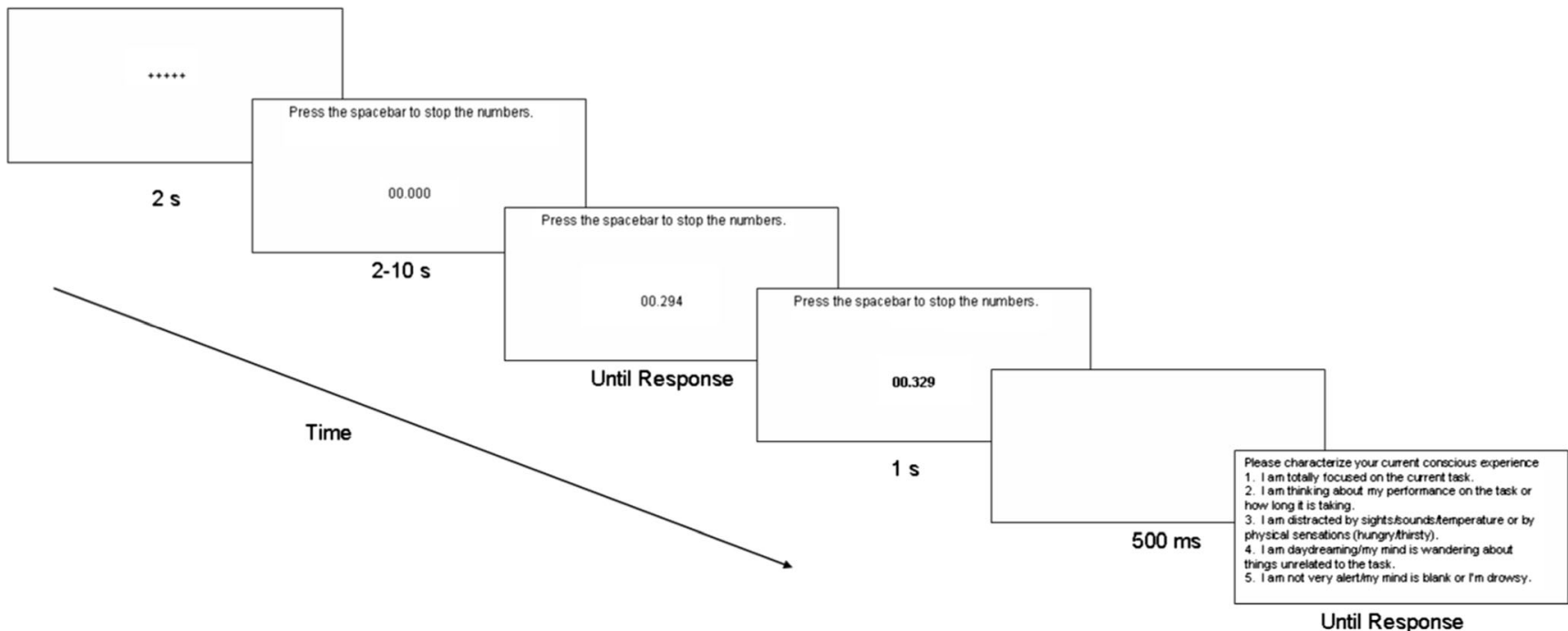

Fig. 1 Schematic of the experimental task with a thought-probe after the trial

Please characterize your current conscious experience.

1. I am totally focused on the current task.

2. I am thinking about my performance on the task or how long it is taking.

3. I am distracted by sights/sounds/temperature or by physical sensations (hungry/thirsty).

4. I am daydreaming/my mind is wandering about things unrelated to the task.

5. I am not very alert/my mind is blank or I'm drowsy.

These thought probes were based on those used by Stawarczyk et al. (2011) and Unsworth and McMillan (2014a). Participants were given specific instructions regarding the different categories based on prior research (McVay \& Kane, 2012b; Stawarczyk et al., 2011; Unsworth \& McMillan, 2014a). Similar to prior research, response three was classified as external distraction and response four was classified as mind-wandering. Response five was classified as inattentive/ nonalert. Response one was considered as on-task thoughts, while response two was considered as task-related interference.

\section{Data Analysis}

Data from each participant's left eye was used (left and right eye pupil diameter were highly correlated, $r=.89$ ). Missing data points due to blinks, off-screen fixations, and/or eyetracker malfunction were removed (roughly $15.6 \%$ of the overall data with roughly equal amounts of data loss across blocks and conditions). The relevant period of missing data was not included in the averaging. Pretrial baseline responses were computed as the average pupil diameter during the fixation screen $(2,000 \mathrm{~ms})$. Pretrial baselines were $z$-scored normalized within each participant to correct for individual differences in pupil diameter. TEPRs were corrected by subtracting out the last $200 \mathrm{~ms}$ of the wait time and locked to when the numbers began counting up on a trial-by-trial basis for each participant. To examine the time course of the TEPRs, the pupil data were averaged into a series of $20 \mathrm{~ms}$ time windows following stimulus onset for each trial.

\section{Results}

\section{Behavioral Results}

Reaction Time (RT) First we examined time on task effects. RTs were grouped into five blocks of 32 trials each. Consistent with prior research a classic vigilance decrement was observed such that as time on task increased so did reaction times (see Figure 2a). There was a main effect of block, $F(4,144)=$ 26.83, $M S E=480.4, p<.01$, partial $\eta^{2}=.43$, and a significant linear trend, $F(1,36)=54.30, M S E=945.89, p<.01$, partial $\eta^{2}=.60$. Additionally, as might be expected the mean standard deviation of the RTs also increased across blocks (see Figure 2b), suggesting that not only did participants get slower across blocks but they also became more variable in their responding. There was a main effect of block, $F(4,144)=$ 8.50, MSE $=747.3, p<.01$, partial $\eta^{2}=.19$, and a significant linear trend, $F(1,36)=26.68, M S E=934.92, p<.01$, partial $\eta^{2}=.43$. Note that we also computed coefficient of variation across blocks to see if variability increased after taking into account differences in the mean. Similar to the analysis on mean standard deviations, mean coefficient of variation increased across blocks, $F(4,144)=5.73, M S E=.004$, $p<.01$, partial $\eta^{2}=.14$. Next, we examined whether lapses of attention would increase with time on task. Lapses of attention were defined as the slowest $20 \%$ of reaction times. Specifically, we ranked ordered each participant's RTs from 

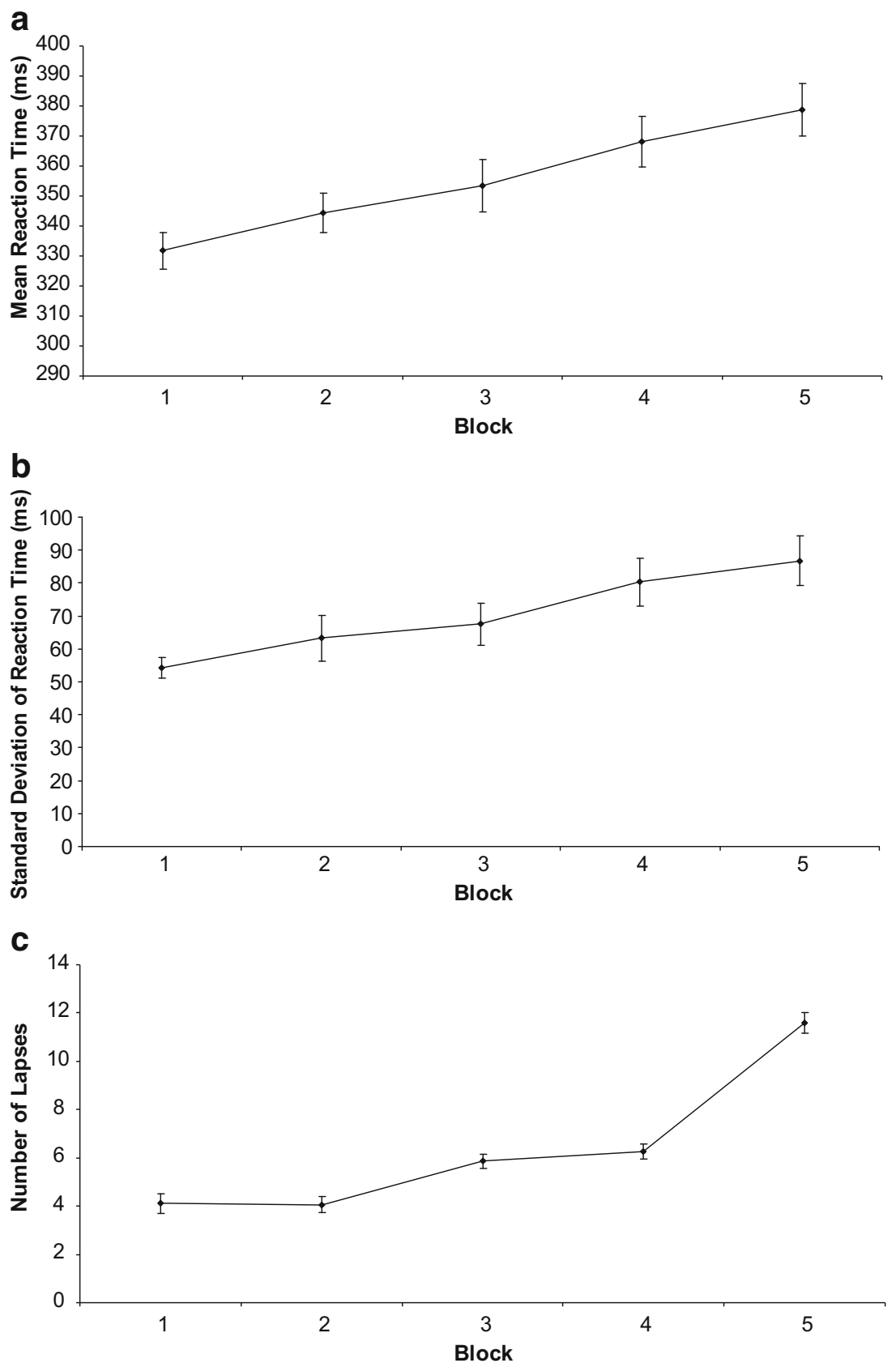

Fig. 2 a Mean reaction time (RT) as a function of block. b Mean standard deviation of reaction time as a function of block. $\mathbf{c}$ Mean number of lapses of attention as a function of block. Error bars reflect 1 standard error of the mean

fastest to slowest and placed into quintiles. The slowest quintile was taken as lapses. This measure has been used previously in many prior individual differences studies to examine how lapses of attention correlate with other cognitive abilities (e.g., Unsworth et al., 2010). As shown in Figure 2c, the number of lapses of attention steadily increased with time on task, with most lapses occurring in the final block. There was a main effect of block, $F(4,144)=58.34, M S E=6.03, p<.01$, partial $\eta^{2}=.62$, and a significant linear trend, $F(1,36)=$ 103.63, $M S E=10.55, p<.01$, partial $\eta^{2}=.74$. Thus, consistent with previous work in sustained attention and vigilance
(Kribbs \& Dinges, 1994; Parasuraman, 1986; Unsworth et al., 2010), the current results suggested that individuals became slower, more variable, and experienced more lapses of attention as time on the task increased.

Thought Probes Next, we examined the thought-probe responses. There was a significant effect of thought probe response, $F(4,144)=4.59, M S E=.054, p<.01$, partial $\eta^{2}=.12$. Shown in Table 1 are the proportions of each thought-probe response. As can be seen, participants spent much of their time either focused on the task or thinking about their performance 
Table 1 Proportions of each thought-probe response

\begin{tabular}{lllll}
\hline On-task & TRI & ED & MW & Inattn \\
\hline $.28(.27)$ & $.27(.19)$ & $.08(.10)$ & $.17(.17)$ & $.20(.26)$ \\
\hline
\end{tabular}

On-task on-task thoughts, TRI task-related interference, $E D$ external distraction, $M W$ mind-wandering, Inattn inattentive/nonalert. Standard deviations are in parentheses

on the task (task-related interference). At the same time, there was considerable off-task thinking with participants reporting that they experienced external distraction, mind-wandering, and inattentiveness.

As shown in Figure 3a, examining the thought probes responses by block suggested a Block $\times$ Thought Probe response interaction, $F(4,144)=4.99, M S E=1.88, p<.01$, partial $\eta^{2}=.12$, such that on-task reports tended to decrease across blocks (linear trend, $p=.012$, partial $\eta^{2}=.16$ ), but reports of mind-wandering (linear trend, $p<.01$, partial $\eta^{2}=.32$ ) and inattentiveness (linear trend, $p<.01$, partial $\left.\eta^{2}=.37\right)$ tended to increase across blocks. External distraction tended to increase across blocks as well (linear trend, $p=.017$, partial $\left.\eta^{2}=.15\right)$. Task-related interference tended to remain fairly constant across blocks (linear trend, $p=.23$, partial $\left.\eta^{2}=.04\right)$. Thus, as time on task increased, participants reported being less focused on the task and reported more off-task thoughts consistent with prior research (e.g., McVay \& Kane, 2012a; Thomson, Seli, Besner, \& Smilek, 2014).

Examining RTs for the different responses suggested that the different off-task reports were associated with much slower RTs than on-task reports, $t=9.24, p<.001$ ( $b=17.75, S E=1.92)$. Note, because not all participants reported all of the attentional states, we used linear mixed models to analyze the data. Linear mixed models are an extension of the general linear model in which both fixed and random effects are included. Thus, they are similar to mixed analysis of variance (ANOVA) but offer advantages over traditional mixed ANOVAs in terms of more power and the ability to handle unbalanced designs and missing data (e.g., Kliegl, Wei, Dambacher, Yan, \& Zhou, 2011). In the model, attentional state was entered as a fixed factor, and subjects were entered as random factors. Note, there were 28 participants contributing to the on-task category, 34 contributing to task-related interference, 26 to external distraction, 31 to mind-wandering, and 23 to the inattentive category. Examining attentional state as fixed factor suggested differences in RTs across the different states, $t=9.10, p<.001$ $(b=8.77, S E=.93)$. Specifically, as shown in Figure $3 b$, comparing trials associated with each off-task thought report to on-
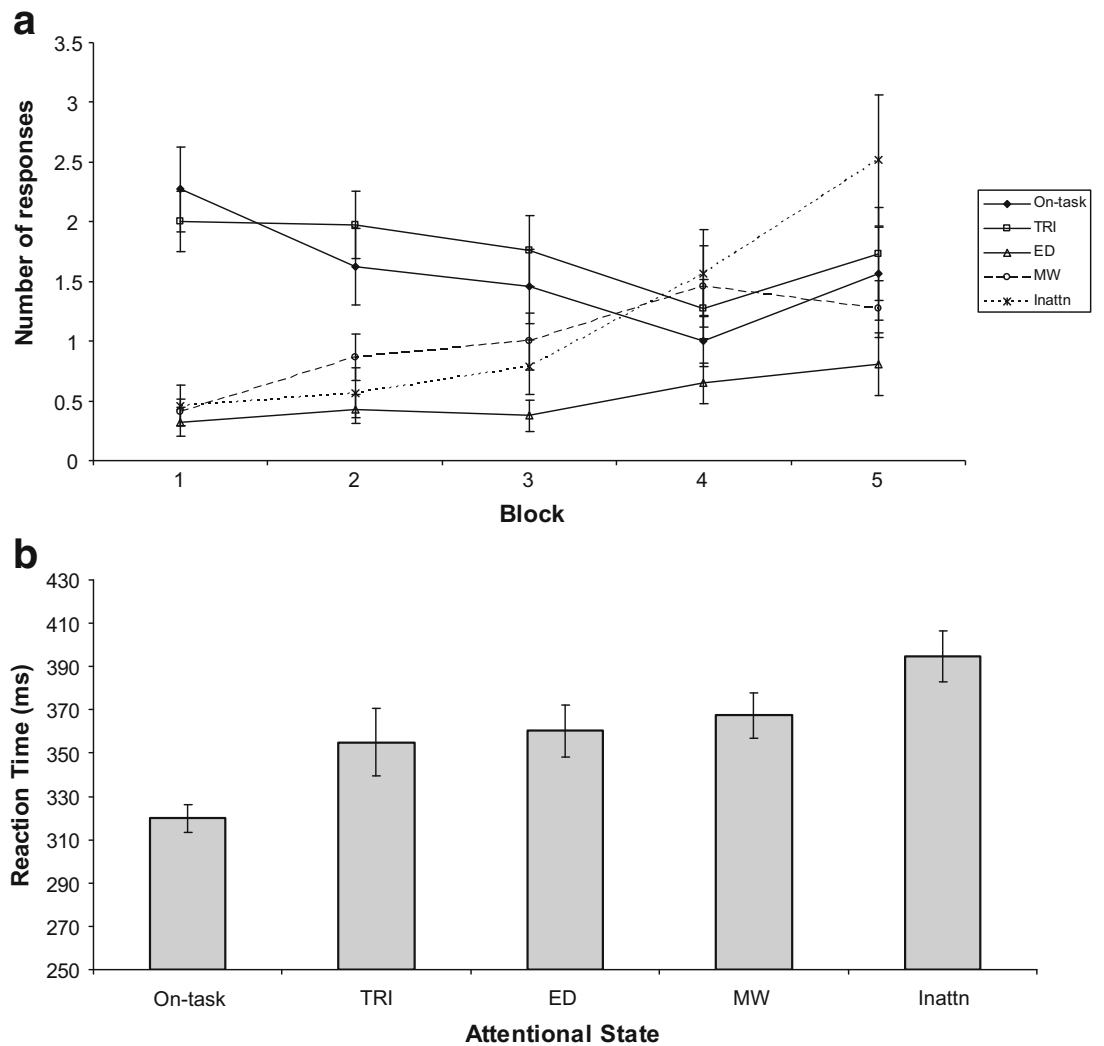

Fig. 3 a Number of thought-probe responses as a function of attentional state and block. b Mean reaction time as a function of attentional state. Error bars reflect 1 standard error of the mean. Note. On-task= on-task thoughts;
TRI = task-related interference; $\mathrm{ED}=$ external distraction; $\mathrm{MW}=$ mindwandering; Inattn $=$ inattentive/nonalert 

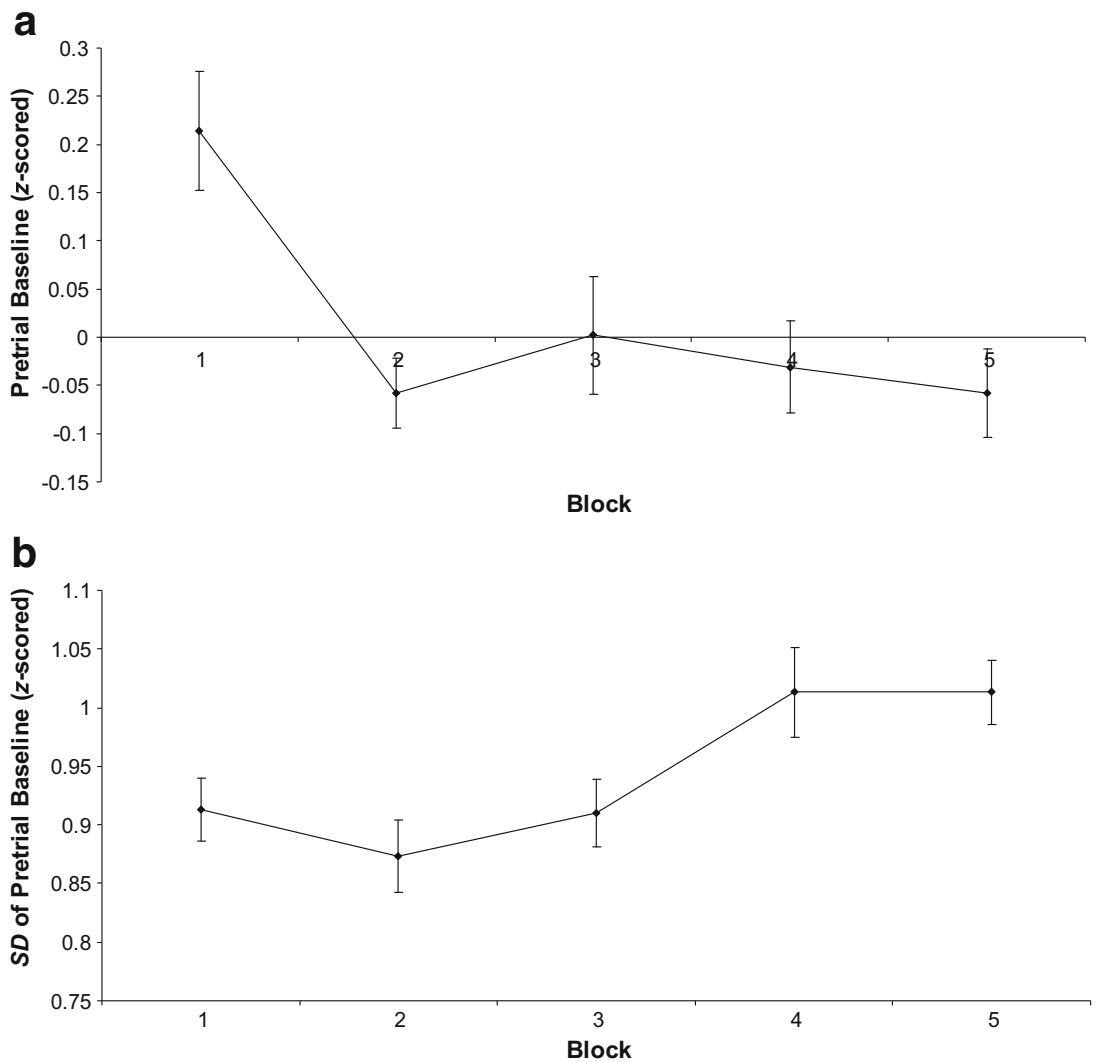

Fig. 4 a Normalized mean pretrial baseline pupil diameter as a function of block. b Mean standard deviation of normalized pretrial baseline pupil diameter. Error bars reflect one standard error of the mean

task thought reports in separate linear mixed models suggested that off-task reports were 40 to $70 \mathrm{~ms}$ slower than trials where participants reported being fully on task (all $p \mathrm{~s}<.01$ ). Thus, when participants reported being distracted, mind-wandering, or being inattentive, performance was slower than when they reported being on task.

\section{Pupil Diameter}

Next, we turn to our primary analyses of interest, examining pupil size. As noted previously, pupil diameter was measured continuously throughout the task. Therefore, both pretrial baseline and TEPRs were examined.

Pretrial Pupil Diameter First, we examined time-on-task effects for pretrial pupil diameter. As shown in Figure 4a, pretrial pupil diameter decreased throughout the task, with the largest decrease happening from Block 1 to Block 2. There was a main effect of block, $F(4,144)=4.08, M S E=.12$, $p<.01$, partial $\eta^{2}=.10$, and a significant linear trend, $F(1,36)=4.88, M S E=.20, p=.034$, partial $\eta^{2}=.34$. This is consistent with prior research, which has shown a vigilance

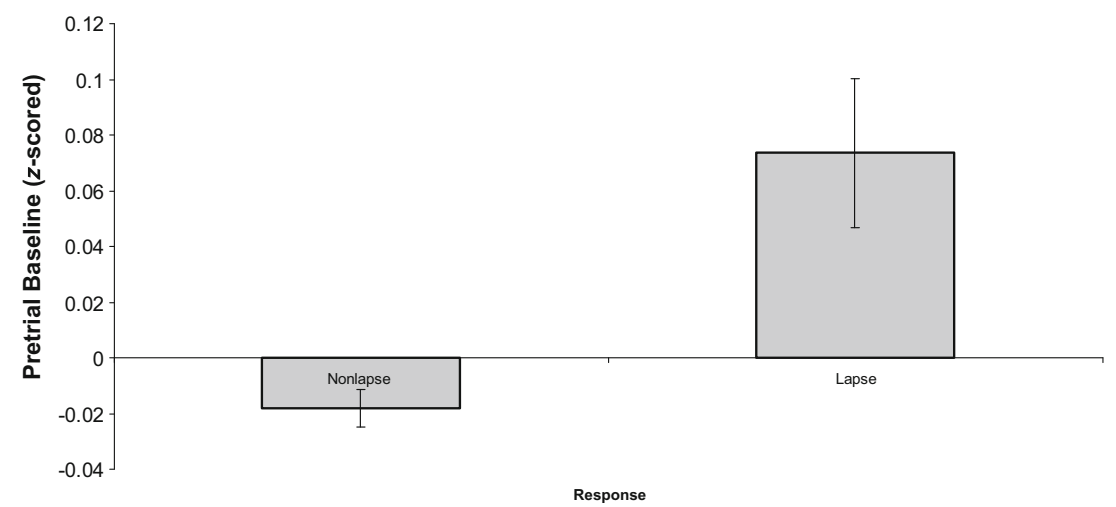

Fig. 5 Normalized mean pre-trial baseline pupil diameter as a function of non-lapse or lapse trials. Error bars reflect one standard error of the mean 
decrement in baseline pupil diameter (Fried et al., 2014; but see Beatty 1982b). Additionally, as seen in Figure 4b, pretrial baseline pupil diameter tended to become more variable with time on task. There was a main effect of block, $F(4,140)=$ $3.75, M S E=.04, p<.01$, partial $\eta^{2}=.10$, and a significant linear trend, $F(1,35)=9.30, M S E=.01, p<.01$, partial $\eta^{2}=.21$. Overall, the decrease in pupil diameter and the increase in pupil variability are consistent with increases in pupillary unrest as time on task increases, suggesting that as time on task increased participants alertness and arousal decreased (Morad et al., 2000; Wilhelm et al., 2001).

Next, we examined pretrial pupil diameter for lapse and nonlapse trials. Specifically, we examined the normalized pretrial baseline pupil diameter for trials classified as a lapse (20\% slowest trails) compared to nonlapse trials (average of the other $80 \%$ of trials). As shown in Figure 5a, pretrial baseline pupil diameter was slightly larger for trials that experienced a subsequent lapse of attention compared to nonlapse trials, $t(36)=$ 2.73, $p=.01, d=.45$. Similar results were obtained when comparing quintile 5 to each of the other quintiles separately (all $p \mathrm{~s}<.046$ ). Thus, when participants had a long RT, these trials were preceded by larger than normal pretrial pupil diameters, consistent with the notion that participants were experiencing task disengagement (Gilzenrat et al., 2010).

Turning to one of the most important analyses, examining pretrial baseline pupil diameter as a function of attentional state suggested clear differences. Again because not everyone reported every attentional state, we used mixed models to analyze the data. Attentional state was entered as a fixed factor, and subjects were entered as random factors. First, examining on-task reports versus off-task reports (i.e., combining external distraction, mind-wandering, and inattentive) suggested that on-task reports $(M=.06, S E=.06)$ were associated with larger pretrial baselines than were off-task reports $(M=-.08, S E=.04), t=-1.97, p=.049(b=-.14, S E=.07)$. This replicates prior work by Grandchamp et al. (2014) and Mittner et al. (2014) but is inconsistent with Franklin et al. (2013). Perhaps the differences are due to differences in the types of lapses that can be considered as off task. To examine this, we analyzed pretrial baseline pupil diameter as a function of the different attentional states. The analysis suggested a main effect of attentional state, $t=-3.21, p<.001(b=-.06$, $S E=.02) .{ }^{1}$ As shown in Fig. 6a and b, pretrial baseline pupil diameter clearly distinguished the different forms of off-task

\footnotetext{
${ }^{1}$ It is possible that differences in pupil size across the different attentional states are due to eye movements. Eye movements were defined as any movements that deviated from center fixation by more than $20 \%$. There were no differences across the attentional states in terms of number of eye movements $(p=.79)$, average eye position $(p=.68)$, or absolute distance from fixation $(p=.80)$ given that most of the time participants were staring in the center of the screen where the stimuli were presented.
}

thought from one another and from on-task thoughts. Specifically, when participants reported being externally distracted, their baseline pupil diameters were much larger than normal (i.e., compared to the overall average baseline pupil diameter; $p<.01$ ), and tended to be larger than when participants reported being on task $(p=.059)$. When participants reported either mind-wandering or being inattentive, their baseline pupil diameters were much smaller than the overall average baseline pupil diameter and much smaller than when participants reported being on task (both $p \mathrm{~s}<.01$ ). Furthermore, both mind-wandering and inattentive reports were associated with smaller pretrial baseline pupil diameters than reports of external distraction (both $p$ s $<.01$ ), but pretrial pupil diameter for mind-wandering and inattentive reports were not different $(p=.55)$. These results demonstrate that different types of lapses of attention are associated with different pretrial baseline pupil levels and further suggest that these lapses are indexing different parts of the LC-NE curve. ${ }^{2}$

Task-Evoked Pupillary Response Our final set of analyses focused on TEPRs to see if the TEPRs would decrease with time on task consistent with the decrease seen in pretrial baseline and consistent with prior research (e.g., Beatty, 1982b). The TEPRs were corrected by subtracting out pupil diameter from the last $200 \mathrm{~ms}$ of the wait time and locked to when the numbers began counting up. Across all trials and participants there were a minimum of 700 data points per bin. Shown in Figure 7 are the TEPRs as a function of block. Note that the pupil waveforms are used mainly for visualization, whereas the dependent measure in the analysis is the peak task-evoked response. There was a clear reduction in the peak task-evoked response as a function of block. There was a main effect of block, $F(4,140)=12.25, M S E=.001, p<.01$, partial $\eta^{2}=.26$, and a significant linear trend, $F(1,35)=33.06, M S E=.001$, $p<.01$, partial $\eta^{2}=.49$. Thus, consistent with prior research, TEPRs decreased with time on task (Beatty, 1982b).

Next, we examined whether TEPRs would change as a function of attentional state. Specifically, on-task responses should be associated with larger phasic responses than the various off-task responses. As shown in Figure 8a, this was indeed the case, with the different off-task responses exhibiting smaller phasic responses than on-task reports. Similar to the block phasic response, the TEPRs were corrected by subtracting out pupil diameter from the last 200

\footnotetext{
${ }^{2}$ Given that there were also effects of block on pretrial baseline pupil and given that off-task thought reports increased as a function of block, we examined whether the differences in attentional state would remain after including block into the model as a covariate. The main effect of attentional state remained $(t=-2.76, p=.004)$, and the interaction between attentional state and block was not quite significant $(t=-1.66, p=.097)$ suggesting that although pretrial pupil diameter changed as a function of attentional state and time on task (see discussion), the effect of attentional state was not exclusively mediated by time-on-task effects.
} 


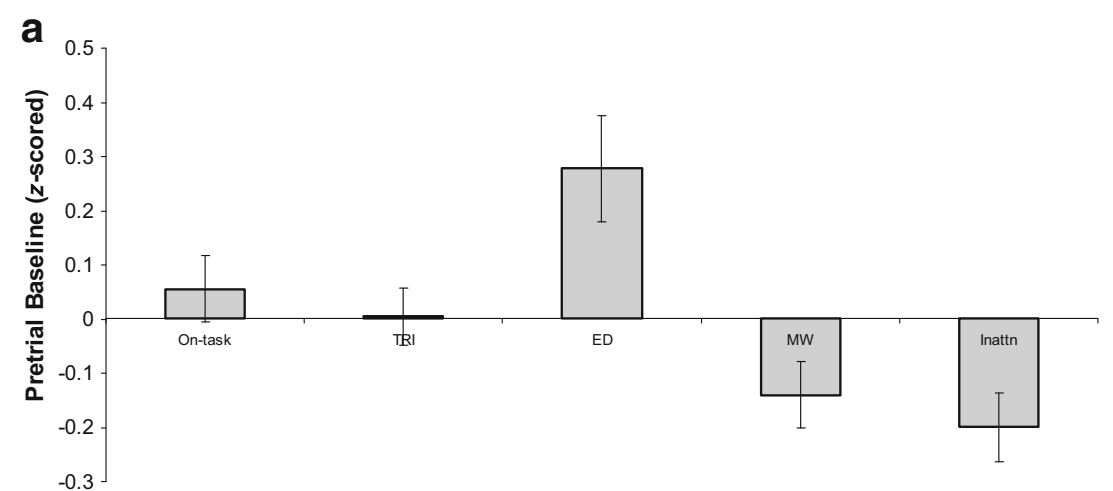

Attentional State

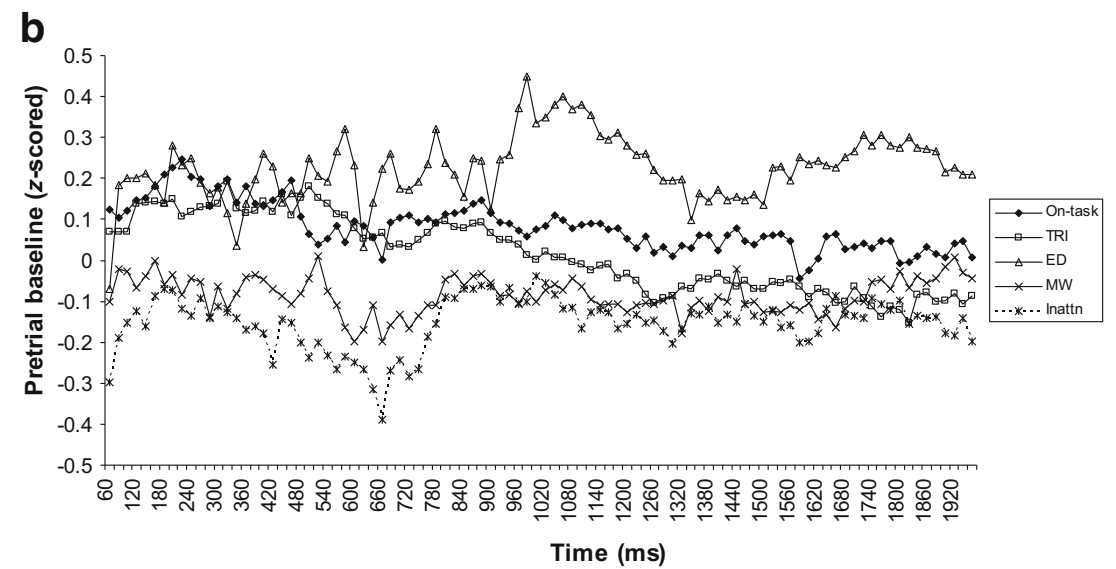

Fig. 6 a Normalized mean pretrial pupil diameter as a function of attentional state. b Normalized mean pretrial pupil diameter as a function of attentional state and time. Error bars reflect 1 standard error of the mean.

ms of the wait time and locked to when the numbers began counting up. As shown in Figure 8b, the peak response differed as a function of attentional state, $t=-2.67, p=.008$ $(b=-.01, S E=.003)$. Specifically, when comparing TEPRs for on-task reports vs. off-task reports (i.e., combining external distraction, mind-wandering, and inattentive), it was suggested that on-task reports were associated with larger phasic responses than off-task reports, $t=-2.66, p=.008(b=-.03$, $S E=.01)$. To examine these further, separate linear mixed
Note. On-task $=$ on-task thoughts; TRI $=$ task-related interference; $\mathrm{ED}=$ external distraction; $\mathrm{MW}=$ mind-wandering; Inattn = inattentive/nonalert

models comparing each off-task response to on-task responses were done. The difference between on-task and off-task reports was mainly driven by differences between on-task responses and mind-wandering $(p=.012)$ and inattentiveness $(p=.044)$. The effect did not reach significance for external distraction $(p=.14)$, given larger variability associated with fewer responses. Additionally, there did not seem to be a difference between TEPRs for on-task and task-related interference $(p=.14)$. Overall, these results are consistent with the

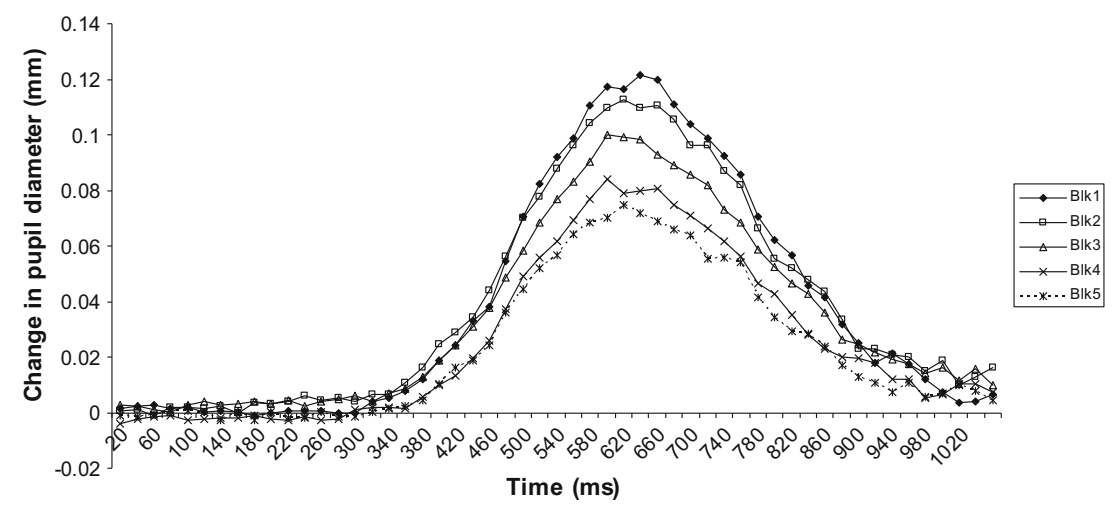

Fig. 7 Task-evoked pupillary responses as a function of block 

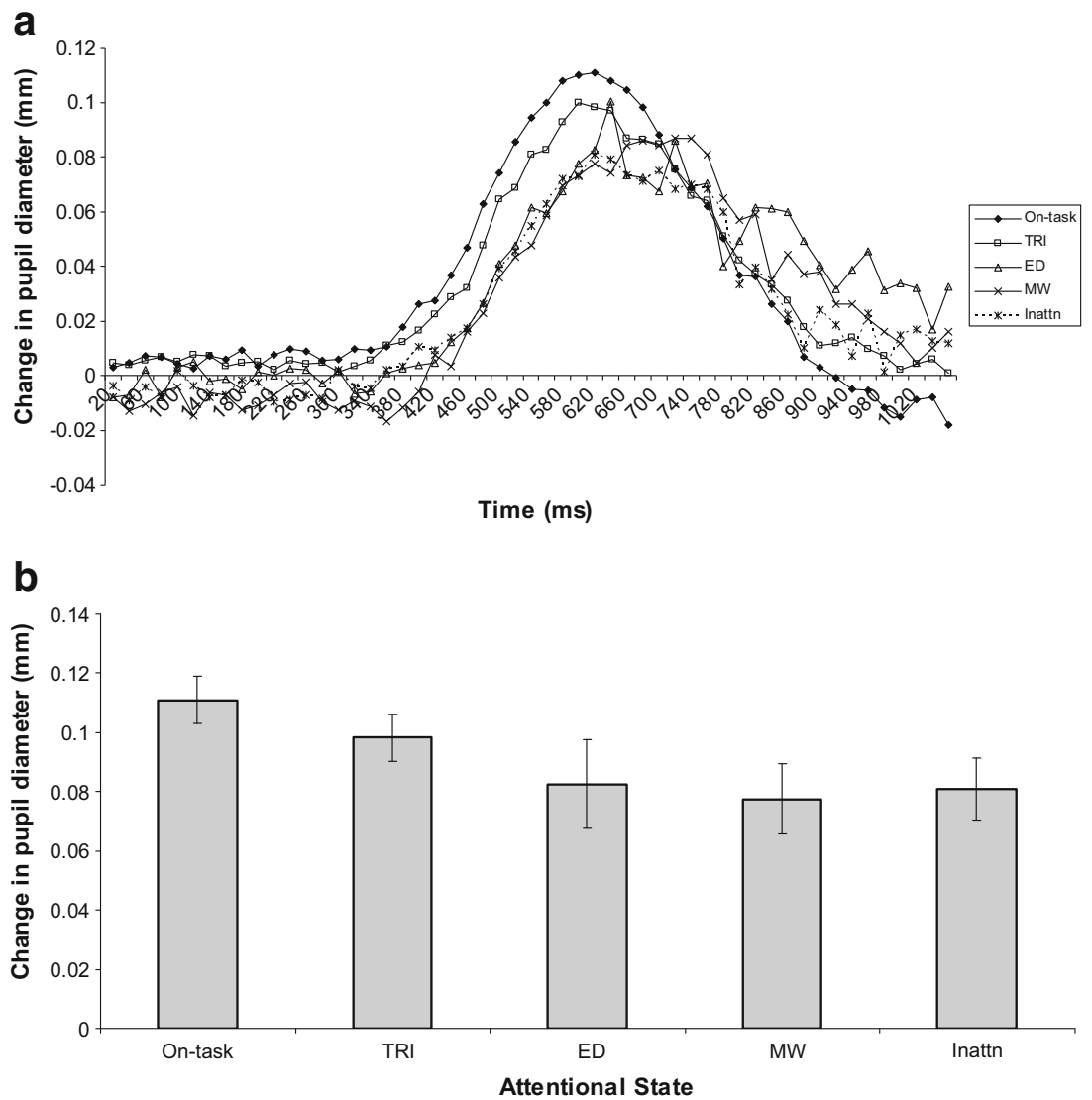

Fig. 8 a Grand averaged task-evoked pupillary responses as a function of attentional state. b Peak response as a function of attentional state. Error bars reflect 1 standard error of the mean. Note. On-task = on-task

notion that unfocused attentional states are associated with reduced TEPRs.

\section{Discussion}

In the current study, we examined whether pupillary responses (both pretrial and phasic) could be used to distinguish between different attentional states. During a sustained-attention task, we found that when participants reported being off task they had smaller than normal pretrial baseline pupil diameters and smaller than normal phasic responses to the stimuli. These results are consistent with some prior research that has similarly shown that when participants are off task both baseline and phasic responses are smaller (e.g., Grandchamp et al., 2014; Mittner et al., 2014). However, the results are inconsistent with prior research by Franklin et al. (2013), who showed that when participants are off task they have larger baseline pupil diameters than when they report being on task. One potential difference between these studies is the tasks used. Both in the current study and in Mittner et al. (2014), participants performed a challenging attention-control task (here, the psychomotor vigilance task and in Mittner et al. a stop- thoughts; TRI = task-related interference; $\mathrm{ED}=$ external distraction; $\mathrm{MW}=$ mind-wandering; Inattn $=$ inattentive $/$ nonalert

signal task), whereas in Franklin et al. (2013) participants read a text. Thus, differences may arise due to differences in how challenging the tasks are, with participants in the attentioncontrol tasks being required to consistently maintain attention on task whereas during a self-paced reading task there may be more opportunities to intentionally disengage from the task. That is, with the self-paced reading task it is possible to take a break and intentionally disengage from the task, resulting in more exploratory behavior thought to be associated with higher LC-NE tonic activity. In the more attentiondemanding tasks, it is necessary to consistently maintain attention on the task given the fast-paced nature of the trials. In these tasks any intentional disengagement can result in much poorer performance. At the same time, given the demand to sustain attention on these tasks, it is possible that as time on task increases, overall arousal decreases, leading to fatigue and lowered alertness. Thus, the different types of tasks may encourage different attentional states associated with different pupillary responses.

Consistent with theories of LC-NE functioning, it is possible that some lapses of attention are a result of lowered arousal and alertness linked to the lower portion of the LC-NE curve. These lapses should be linked with 
poorer behavioral performance, small baseline pupil diameters, and small phasic responses. As suggested by Lenartowicz et al. (2013), depending on whether attention is focused internally or externally, lowered arousal levels can be associated with either mind-wander or external distraction. Additionally, it is possible that other lapses of attention are a result of heightened arousal linked with the upper portion of the LC-NE curve. These lapses should also be linked with poorer behavioral performance and small phasic responses, but, critically, these lapses should be linked with larger than normal baseline pupil diameters. Again, as suggested by Lenartowicz et al. (2013), depending on whether attention is focused internally or externally, heightened arousal levels can be associated with different types of mind-wandering and external distraction. The current results are very much in line with this hypothesis. Specifically, we found that when participants reported being inattentive or mind-wandering, their RTs were longer, their pretrial baseline pupil diameters were smaller, and their phasic responses were smaller than when they reported being on task. Additionally, when participants reported being distracted, their RTs were longer, their pretrial baseline pupil diameters were larger, and their phasic responses were (nonsignificantly) smaller than when they reported being on task. Consistent with recent theorizing, these results strongly suggest that there are different flavors of lapses of attention with each being linked with different modes of LC-NE functioning as indicated by differences in the pupillary responses. These results demonstrate that fluctuations in attentional state can be tracked with changes in pupil dilations and further suggest that changes in pupil dilation can distinguish different types of lapses of attention.

At the same time, the current results suggest that there is likely not a simple one-to-one correspondence between different attentional states and pupil dilation. Rather, depending on the current context, task, and individual, it is likely that different attentional states will be associated with either high or low pupil diameters. It is likely that there is a complex interaction of within- and between-subjects effects. Some participants may have a propensity to shift to high arousal (anxious/ stressed), some may shift to low arousal (inattentive/bored), and still others may shift between different states more readily (more variable overall). Furthermore, these effects may also depend on where you are at on the arousal function to begin with. Thus, additional research is needed to better examine the relation between pupillary responses and attentional states, perhaps via experimental manipulations that force or encourage certain states (anxious, bored, exploratory, etc.). Additionally, future research is needed to better delineate different types of mind-wandering and external distraction as a function of arousal level. One problem with the current study is that we relied on very broad categories of attentional state via the thought probes. This makes it difficult to distinguish between different types of attentional states (i.e., mindwandering when bored vs. mind-wandering when anxious). Future research should rely on more fine-grained probes to better capture the different varieties of lapse of attention and subjective attentional states.

Examining time-on-task effects, we found traditional behavioral effects with average RTs increasing and RT variability increasing with time on task. Furthermore, lapses of attention increased substantially with time on task. We also found that, as time on task increased, on-task reports decreased, while reports of inattention and mind-wandering increased. Thus, as time on task increased, alertness decreased and fluctuations in attention increased, resulting in participants being less able to consistently sustain attention on the task. In terms of pupillary responses, we found that as time on task increased, pretrial baseline pupil diameter decreased and variability in pretrial baseline pupil diameter increased. These results are consistent with prior work on pupillary unrest in which, as alertness and arousal decrease and participants become fatigued, the pupil tends to decrease in size with increasing variability (Morad et al., 2000; Wilhelm et al., 2001). Consistent with prior work, we also found that lapses of attention were associated with larger than normal pretrial baseline pupils on average, suggesting that as time on task increased, participants periodically disengaged from the task (Gilzenrat et al., 2010). Thus, time on task led to overall lowered alertness levels and more fluctuations between the different LC-NE modes. Finally, examining phasic pupillary responses, we found that as time on task increased, phasic pupillary responses decreased (Beatty, 1982b).

Collectively, the current results are consistent with prior LC-NE research, which has shown that during periods of lowered vigilance, tonic LC activity is reduced and phasic LC responses are diminished, leading to poorer behavioral performance consistent with lapses of attention (Aston-Jones et al., 1994; Aston-Jones, Iba, Clayton, Rajkowski, \& Cohen, 2007). That is, as time on task increases, alertness and arousal decrease, making it increasingly difficult to sustain attention on task. With these decreases in arousal and alertness, attention fluctuates widely between different states. Subjectively, this results in participants reporting more inattention, mindwandering, and distraction, as it is increasingly difficult to maintain focus on the current task and prevents thoughts from drifting to unrelated matters. These increases in lapses of attention lead to very slow and to more inconsistency in reaction times. Importantly the current results suggest that pupillary changes accurately track these changes in fluctuations in attentional state in a manner consistent with prior LC-NE theorizing (e.g., Aston-Jones \& Cohen, 2005; Gilzenrat et al., 2010; Jepma \& Nieuwenhuis, 2011; Murphy, Robertson, Balsters, \& O'Connell, 2011). As such, the current results provide important information - not only on changes in 
sustained attention as time on task increases but also on the notion that there are different flavors of lapses of attention associated with different pupillary responses, theoretically due to different modes of LC-NE system. The current results not only inform theories of sustained attention and lapses of attention but also provide an important tool for tracking and distinguishing various types of lapses of attention. Thus, pupillometry provides the potential for an online unobtrusive and objective marker of fluctuations in attentional state that can be used to predict when lapses of attention are about to occur and potentially intervene and interrupt or correct those lapses. Future work is needed to further examine the extent to which pupillary responses accurately track different types of lapses of attention in a variety of situations and contexts and to examine the feasibility of using pupillometry as an online marker of fluctuations in attentional state.

\section{References}

Alnaes, D., Sneve, M. H., Espeseth, T., Endestad, T., van de Pavert, S. H. P., \& Laeng, B. (2014). Pupil size signals mental effort deployed during multiple object tracking and predicts brain activity in the dorsal attention network and the locus coeruleus. Journal of Vision, 14, 1-20.

Aston-Jones, G., \& Cohen, J. D. (2005). An integrative theory of locus coeruleus-norepinephrine function: Adaptive gain and optimal performance. Annual Review of Neuroscience, 28, 403-450.

Aston-Jones. G., Rajkowski, J., Kubiak, P., Alexinsky, T. (1994). Locus coeruleus neurons in the monkey are selectively activated by attended stimuli in a vigilance task. The Journal of Neuroscience, 14, 4467-4480.

Aston-Jones, G. S., Iba, M., Clayton, E., Rajkowski, J., \& Cohen, J. (2007). The locus coeruleus and regulation of behavioral flexibility and attention: Clinical implications. In G. A. Ordway, M. A. Schwartz, \& A. Frazer (Eds.), Brain norepinephrine: Neurobiology and therapeutics (pp. 196-235). Cambridge: Cambridge University Press.

Beatty, J. (1982a). Task-evoked pupillary responses, processing load, and the structure of processing resources. Psychological Bulletin, 91, 276-292.

Beatty, J. (1982b). Phasic not tonic pupillary responses vary with auditory vigilance performance. Psychophysiology, 19, 167-172.

Beatty, J., \& Lucero-Wagoner, B. (2000). The pupillary system. In J. T. Cacioppo, L. G. Tassinary, \& G. G. Berntson (Eds.), Handbook of psychophysiology (pp. 142-162). New York: Cambridge University Press.

Berridge, C. W., \& Waterhouse, B. D. (2003). The locus coeruleusnoradrenergic system: Modulation of behavioral state and statedependent cognitive processes. Brain Research Reviews, 42, 33-84.

Cohen, J. D., Aston-Jones, G., \& Gilzenrat, M. S. (2004). A systems-level perspective on attention and cognitive control: Guided activation, adaptive gating, conflict monitoring, and exploitation vs. exploration. In M. I. Posner (Ed.), Cognitive neuroscience of attention (pp. 71-90). New York: Guilford Press.

Dinges, D. F., \& Powell, J. W. (1985). Microcomputer analyses of performance on a portable, simple visual RT task during sustained operations. Behavior Research Methods, Instruments, \& Computers, 17, 652-655.
Dorrian, J., Rogers, N. L., \& Dinges, D. F. (2005). Psychomotor vigilance performance: A neurocognitive assay sensitive to sleep loss. In C. Kushida (Ed.), Sleep deprivation: Clinical issues, pharmacology and sleep loss effects. New York: Marcel Dekker.

Drummond, S. P. A., Bischoff-Grethe, A., Dinges, D. F., Ayalon, L., Mednick, S. C., \& Meloy, M. J. (2005). The neural basis of the psychomotor vigilance task. Sleep, 28, 1059-1068.

Duncan, J. (1995). Attention, intelligence, and the frontal lobes. In M. Gazzaniga (Ed.), The cognitive neurosciences (pp. 721-733). Cambridge: MIT Press.

Franklin, M. S., Broadway, J. M., Mrazek, M. D., Smallwood, J., \& Schooler, J. W. (2013). Window to the wandering mind: Pupillometry of spontaneous thought while reading. The Quarterly Journal of Experimental Psychology, 1-15.

Fried, M., Tsitsiashvili, E., Bonney, Y. S., Sterkin, A., Wygnanski-Jaffe, T., Epstein, T., \& Polat, U. (2014). ADHD subjects fail to suppress eye blinks and mircosaccades while anticipating visual stimuli but recover with medication. Vision Research, 101, 62-72.

Gilzenrat, M. S., Nieuwenhuis, S., Jepma, M., \& Cohen, J. D. (2010). Pupil diameter tracks changes in control state predicted by the adaptive gain theory of locus coeruleus function. Cognitive, Affective, \& Behavioral Neuroscience, 10, 252-269.

Grandchamp, R., Braboszcz, C., \& Delorme, A. (2014). Occulometric variations during mind wandering. Frontiers in Psychology, 5, 31.

Hess, E. H., \& Polt, J. M. (1964). Pupil size in relation to mental activity during simple problem-solving. Science, 143, 1190-1192.

Hou, R. H., Freeman, C., Langley, R. W., Szabadi, E., \& Bradshaw, C. M. (2005). Does modafinil activate the locus coeruleus in man? Comparison of modafinil and clonidine on arousal and autonomic functions in human volunteers. Psychopharmacology, 181, 537549.

Jepma, M., \& Nieuwenhuis, S. (2011). Pupil diameter predicts changes in the exploration-exploitation trade-off: Evidence for the adaptive gain theory. Journal of Cognitive Neuroscience, 23, 1587-1596.

Joshi, S., Li, Y., Kalwani, R. M., \& Gold, J. I. (2016). Relationship between pupil diameter and neuronal activity in the locus coeruleus, colliculi, and cingulate cortex. Neuron. doi:10.1016/j.neuron.2015. 11.028 .

Kahneman, D. (1973). Attention and effort. Upper Saddle River: Prentice Hall.

Kahneman, D., \& Beatty, J. (1966). Pupil diameter and load on memory. Science, 154, 1583-1585.

Kang, O. E., Huffer, K. E., \& Wheatley, T. P. (2014). Pupil dilation dynamics track attention to high-level information. PLOS ONE, 9, e102463.

Kliegl, R., Wei, P., Dambacher, M., Yan, M., \& Zhou, X. (2011). Experimental effects and individual differences in Linear Mixed Models: Estimating the relationship between spatial, object, and attraction effects in visual attention. Frontiers in Psychology, 1, 238.

Kribbs, N. B., \& Dinges, D. (1994). Vigilance decrements and sleepiness. In R. D. Ogilvie \& J. R. Harsh (Eds.), Sleep onset: Normal and abnormal processes. Washington, DC: American Psychological Association Press.

Kristjansson, S. D., Stern, J. A., Brown, T. B., \& Rohrbaugh, J. W. (2009). Detecting phasic lapses of alterness using pupillometric measures. Applied Ergonomics, 40, 978-986.

Lenartowicz, A., Simpson, G. V., \& Cohen, M. S. (2013). Perspective: Causes and functional significance of temporal variations in attention control. Frontiers in Human Neuroscience, 7, 381.

McVay, J. C., \& Kane, M. J. (2012a). Drifting from slow to "D'oh!" Working memory capacity and mind wandering predict extreme reaction times and executive-control errors. Journal of Experimental Psychology: Learning, Memory, and Cognition, 38, $525-549$.

McVay, J. C., \& Kane, M. J. (2012b). Why does working memory capacity predict variation in reading comprehension? On the influence 
of mind wandering and executive attention. Journal of Experimental Psychology: General, 141, 302-320.

Miller, E. K., \& Cohen, J. D. (2001). An integrative theory of prefrontal cortex functioning. Annual Review of Neuroscience, 24, 167-202.

Mittner, M., Boekel, W., Tucker, A., Turner, B. M., Heathcote, A., \& Forstmann, B. U. (2014). When the brain takes a break: A modelbased analysis of mind wandering. The Journal of Neuroscience, 34, 16286-16295.

Morad, Y., Lemberg, H., Yofe, N., \& Dagan, Y. (2000). Pupillography as an objective indicator of fatigue. Current Eye Research, 21, 535542 .

Murphy, P. R., O'Connell, R. G., O'Sullivan, M., Robertson, I. H., \& Balsters, J. H. (2014). Pupil diameter covaries with BOLD activity in human locus coeruleus. Human Brain Mapping, 35, 4140-4154.

Murphy, P. R., Robertson, I. H., Balsters, J. H., \& O'Connell, R. G. (2011). Pupillometry and P3 index the locus coeruleusnoradrenergic arousal function in humans. Psychophysiology, 48, $1532-1543$

Nieuwenhuis, S., de Geus, E. J., \& Aston-Jones, G. (2011). The anatomical and functional relationship between the $\mathrm{P} 3$ and autonomic components of the orienting response. Psychophysiology, 48, 162-175.

Parasuraman, R. (1986). Vigilance, monitoring, and search. Handbook of perception and human performance. Vol. 2: Cognitive processes and performance. New York: Wiley.

Rajkowski, J., Kubiak, P., \& Aston-Jones, G. (1993). Correlations between locus coeruleus (LC) neural activity, pupil diameter and behavior in monkey support a role of LC in attention. Society for Neuroscience Abstracts, 19, 974.

Reason, J. T. (1984). Lapses of attention in everyday life. In R. Parasuraman \& D. R. Davies (Eds.), Varieties of attention. Orlando: Academic Press.

Reason, J. T. (1990). Human error. Cambridge: Cambridge University Press.

Roberts, R. J., \& Pennington, B. F. (1996). An integrative framework for examining prefrontal cognitive processes. Developmental Neuropsychology, 12, 105-126.

Schooler, J. W., Reichle, E. D., \& Halpern, D. V. (2005). Zoning out while reading: Evidence for dissociations between experience and metaconsciousness. In D. Levin (Ed.), Thinking and seeing: Visual metacognition in adults and children (pp. 203-226). Cambridge: MIT Press.

Smallwood, J., Brown, K. S., Baird, B., Mrazek, M. D., Franklin, M. S., \& Schooler, J. W. (2012). Insulation for daydreams: A role for tonic norepinephrine in the facilitation of internally guided thought. PLOS ONE, 7, e33706.

Smallwood, J., Brown, K. S., Tipper, C., Giesbrecht, B., Franklin, M. S., Mrazek, M. D., ... Schooler, J. W. (2011). Pupillometric evidence for the decoupling of attention from perceptual input during offline thought. PLOS ONE, 6, e18298.

Smallwood, J., \& Schooler, J. W. (2006). The restless mind. Psychological Bulletin, 132, 946-958.

Stawarczyk, D., Majerus, S., Maj, M., Van der Linden, M., \& D'Argembeau, A. (2011). Mind-wandering: Phenomenology and function as assessed with a novel experience sampling method. Acta Psychologica, 136, 370-381.

Thomson, D. R., Seli, P., Besner, D., \& Smilek, D. (2014). On the link between mind-wandering and task performance over time. Consciousness \& Cognition, 27, 14-26.

Unsworth, N., \& McMillan, B. D. (2014a). Similarities and differences between mind-wandering and external distraction: A latent variable analysis of lapses of attention and their relation to cognitive abilities. Acta Psychologica, 150, 14-25.

Unsworth, N., \& McMillan, B. D. (2014b). Trial-to-trial fluctuations in attentional state and their relation to intelligence. Journal of Experimental Psychology: Learning, Memory, \& Cognition, 40, 882-891.

Unsworth, N., McMillan, B. D., Brewer, G. A., \& Spillers, G. J. (2012). Everyday attention failures: An individual differences investigation. Journal of Experimental Psychology: Learning, Memory, \& Cognition, 38, 1765-1772.

Unsworth, N., Redick, T. S., Lakey, C. E., \& Young, D. L. (2010). Lapses in sustained attention and their relation to executive and fluid abilities: An individual differences investigation. Intelligence, 38, 111122.

Unsworth, N., \& Robison, M. K. (2015). Individual differences in the allocation of attention to items in working memory: Evidence from pupillometry. Psychonomic Bulletin \& Review, 3, 757-765.

Unsworth, N., Schrock, J. C., \& Engle, R. W. (2004). Working memory capacity and the antisaccade task: Individual differences in voluntary saccade control. Journal of Experimental Psychology: Learning, Memory, and Cognition, 30, 1302-1321.

Usher, M., Cohen, J. D., Servan-Schreiber, D., Rajkowski, J., \& AstonJones, G. (1999). The role of locus coeruleus in the regulation of cognitive performance. Science, 283, 549-554.

Ward, A. F., \& Wegner, D. M. (2013). Mind-blanking: When the mind goes away. Frontiers in Psychology, 4, 650.

Wilhelm, B., Giedke, H., Ludtke, H., Bittner, E., Hofmann, A., \& Wilhelm, H. (2001). Daytime variations in central nervous system activation measured by a pupillographic sleepiness test. Journal of Sleep Research, 10, 1-7.

Yerkes, R. M., \& Dodson, J. D. (1908). The relation of strength of stimulus to rapidity of habit-formation. Journal of Comparative Neurology and Psychology, 18, 459-482. 